\title{
Myogenic Heartbeat in the Primitive Crustacean Triops longicaudatus
}

\author{
HIROSHI YAMAGISHI*, HITOSHI ANDO†, AND TOSHIKI MAKIOKA \\ Institute of Biological Sciences, University of Tsukuba, Tsukuba, Ibaraki 305, Japan
}

\begin{abstract}
Pacemaker mechanisms in the heart of the primitive crustacean Triops longicaudatus were examined electrophysiologically. The heart is tubular and the heart wall consists of a single layer of myocardial cells. No nerve cells were found in the heart, either with methylene blue vital staining or by light microscopy of serial sections. The heart beats rhythmically at a frequency of 120 to 240 beats $/ \mathrm{min}$, and each beat is associated with a slow membrane potential change in the heart muscle. The amplitude of the slow potential varies widely and no spikes appear on it. The heart muscle cells are electrically coupled with each other and generate synchronous slow potentials. No localized portion of the heart exhibited a frequency that always preceded the others. The muscle activity could be phase-shifted by injection of a single brief current pulse and could be entrained to a lower or higher frequency by repeated bricf current pulses injected into the muscle cell. The frequency of muscle activity could be changed by the injection of DC current into the muscle cell, and the change in frequency was linearly related to the intensity of the current. When the intensity of hyperpolarizing DC current exceeded a certain value, the muscle activity disappeared abruptly, and the heart stopped beating completely. These results show clearly that the heartbeat of Triops is myogenic. The heart is diffusely myogenic and should be regarded as a single muscle oscillator.
\end{abstract}

\section{Introduction}

The heartbeat of many crustaceans is neurogenic (reviewed by Krijgsman, 1952; Maynard, 1960; Prosser,

Received 10 April 1997; accepted 6 October 1997.

* To whom correspondence should be addressed. E-mail: yamagishi @biol.tsukuba.ac.jp

† Present Address: Department of Biomaterials Science. Faculty of Dentistry, Tokyo Medical and Dental University, Yushima, Bunkyoku, Tokyo 113, Japan.
1973). The cardiac ganglion situated in the heart acts as a pacemaker. Neurogenic heart muscle has no endogenous rhythmical properties and is driven by periodic bursting activity of the cardiac ganglion via excitatory neuromuscular junctions. However, some diversity in the pacemaker mechanism of crustacean hearts has been reported recently. Yamagishi (1996) and Yamagishi and Hirose (1997) have shown, in the isopod Ligia exotica, that the heart of embryos and early juveniles exhibits myogenic heartbeats; i.e., the activity in the myocardium arises endogenously. Later, as the juveniles develop, the cardiac ganglion initiates its spontaneous bursting activity and begins to act as a primary pacemaker, entraining the muscle activity via neuromuscular excitatory junctional potentials. This type of late-developing neurogenic heartbeat is different from that in any other known crustacean.

The heartbeat of branchiopods, one of the lower orders within the Crustacea, is said to be myogenic (reviewed by Krijgsman, 1952; Maynard, 1960). This idea was proposed on the basis of the following two observations. First, no nerve cells were found in the heart of Daphnia (Ingle, cited by Prosser, 1942). Second, the heartbeat of Daphnia was suppressed by external application of acetylcholine to the body, which was thought to suppress myogenic heartbeats (Baylor, 1942; Bekker and Krijgsman, 1951). However, the second observation is not now regarded as evidence of a myogenic heartbeat, because acceleration of the myogenic heartbeat by acetylcholine has been reported (e.g., Greenberg, 1965). Thus, the cardiac pacemaker mechanism of branchiopods is still uncertain.

To explore the diversity in the heartbeat pacemaker mechanisms of crustaceans, we examined the electrophysiology of the heart of the primitive crustacean Triops longicaudatus (Notostraca, Branchiopoda). The aim was to determine whether the heartbeat is myogenic. The results show clearly that the heartbeat of Triops is myogenic, and that the heart should be regarded as a single 
muscle oscillator. Some of these observations have appeared in abstract form (Yamagishi et al., 1993).

\section{Materials and Methods}

\section{Animals}

Adult specimens of the freshwater tadpole shrimp Triops longicaudatus have been collected routinely in June from rice fields in Saitama, Japan. They were kept in a freshwater aquarium in the laboratory, at room temperature, for about 3 weeks. Over a hundred specimens, 15 to $26 \mathrm{~mm}$ in body length, were used for the experiments.

\section{Morphology}

Morphological observations of the heart were made in dissected animals by vital staining with methylene blue, or by light microscopy in serial sections of the heart. For sections, hearts that had been isolated together with the dorsal body wall were fixed in aqueous Bouin's solution. Then, the preparations were dehydrated in a graded ethanol- $n$-butanol series, embedded in paraffin, and cut transversely into serial sections ( 5 to $7 \mu \mathrm{m}$ ). The sections were stained for light microscopy with Mayer's hematoxylin and eosin.

\section{Preparation of the intact heart}

The tubular heart of Triops is situated just under the dorsal body wall in the thoracic region. After the legs were removed, the body was opened by a longitudinal incision through the ventral body wall. The digestive and genital organs in the thorax were carefully removed to expose the heart. The head and abdomen were also removed by two transverse cuts through the body at either end of the thorax. Thus, the heart was isolated together with the dorsal body wall to which it was still attached. Then, the heart preparation was placed, ventral side up, in the experimental chamber and was fixed to the bottom by pins through the dorsal body wall. The chamber was perfused with aerated physiological saline solution throughout the experiment. Under these conditions, the heart beat regularly for several hours. The saline was based on the ionic composition of Triops hemolymph (Horne, 1966), and had the following composition ( $\mathrm{mM}$ ): $\mathrm{NaCl}, 75 ; \mathrm{KCl}, 5 ; \mathrm{CaCl}_{2}, 2 ; \mathrm{MgCl}_{2}, 1$; and Tris- $\mathrm{HCl}(\mathrm{pH}$ 7.4), 5. In some cases, tetrodotoxin (TTX; Sigma) was added to the saline at various concentrations up to $10^{-6} \mathrm{M}$.

\section{Electrophysiology}

The membrane potential of the heart muscle cell was recorded with a conventional glass capillary microelectrode filled with $3 \mathrm{M} \mathrm{KCl}$ (resistance; $10-30 \mathrm{M} \Omega$ ). Another microelectrode, for current injection, was also in-
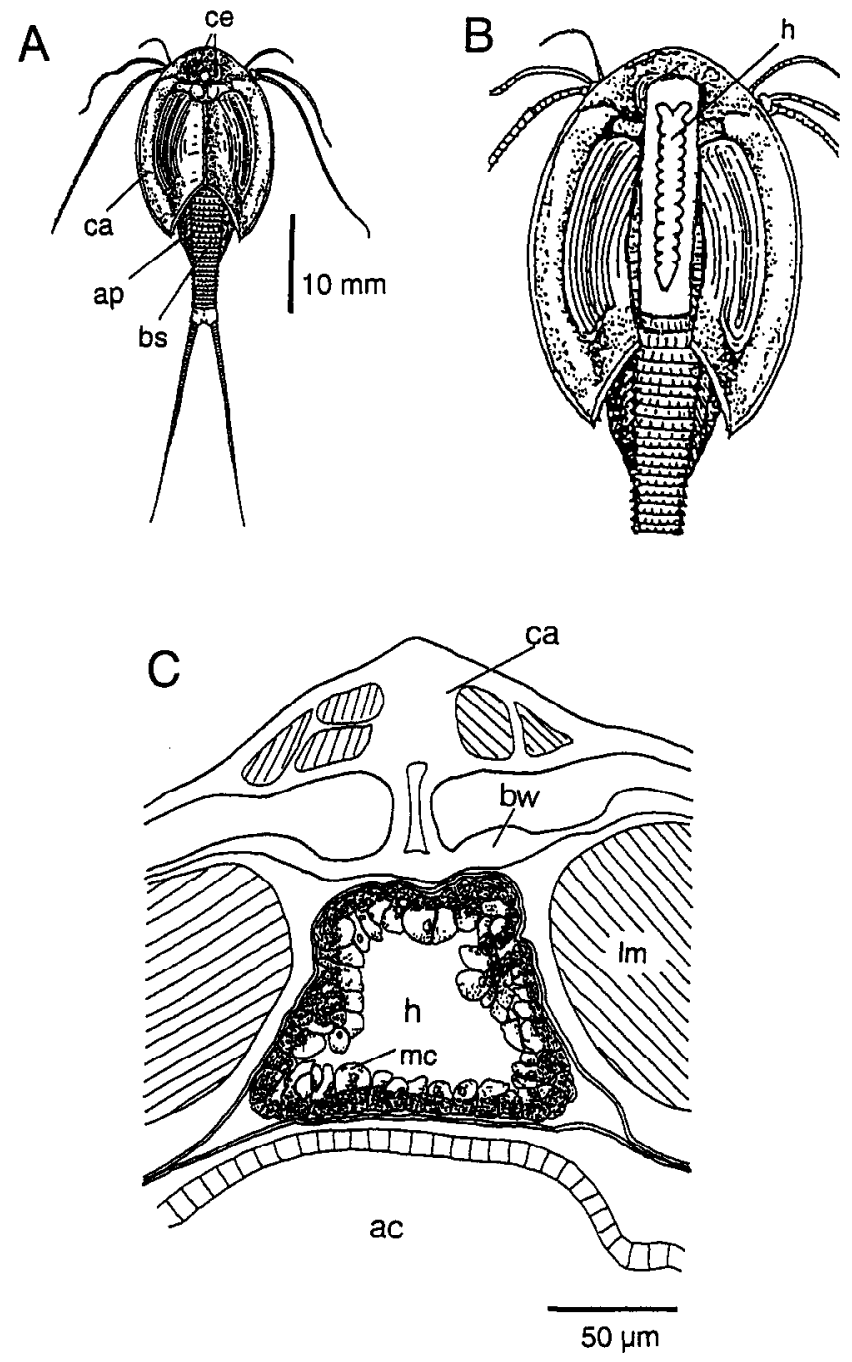

Figure 1. Schematic drawings of the heart of Triops longicaudatus. (A) Dorsal view of the adult Triops. ap, appendage; bs, body segment; ca, carapace; ce, compound eye. (B) Heart exposed by removal of the carapace and the dorsal body wall. h, heart. (C) Transverse section of the body through the thorax. The heart and surrounding tissues are drawn. ac, alimentary canal; bw, body wall; ca, carapace; h, heart; lm, longitudinal muscle; mc, myocardial cell.

serted into the heart muscle cell, but at a distance from the recording electrode. Contraction of the heart was recorded by placing a high-resistance microelectrode lightly on the dorsal heart wall. The data were stored in an FM magnetic tape recorder and were displayed on a cathode ray oscilloscope and a chart recorder. The experiments were carried out at $20^{\circ}$ to $23^{\circ} \mathrm{C}$.

\section{Results}

\section{Anatomy and histology of the heart}

The heart of Triops, a long tubular organ, is situated under the dorsal surface of the body (Fig. 1A, B). It 

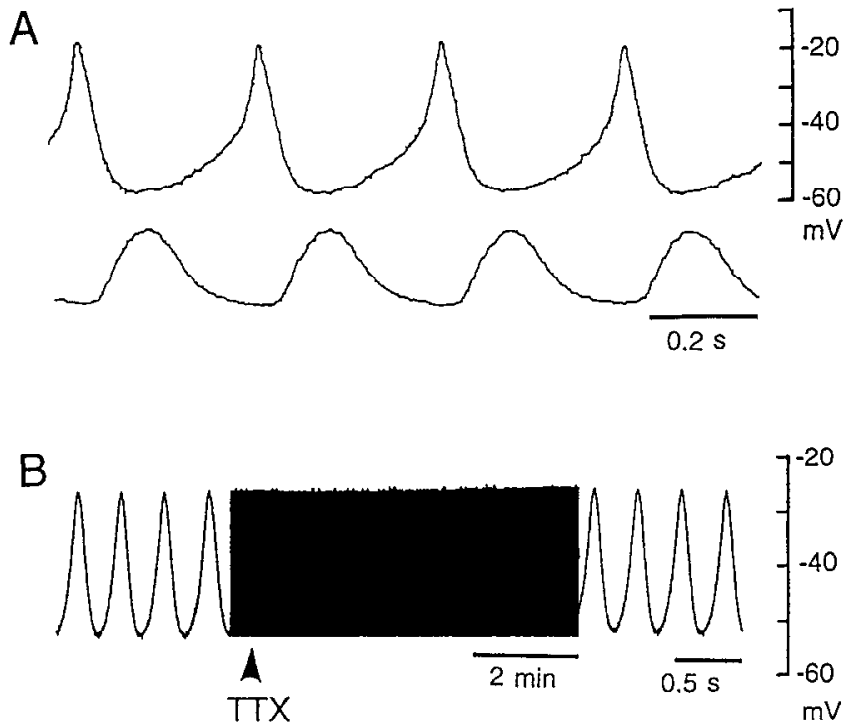

Figure 2. (A) Electrical activity of the heart muscle (upper trace) and contraction of the heart (lower trace) recorded simultaneously. Intracellular membrane potential was recorded from a muscle cell in the anterior region of the heart, and contraction at a site in the central region of the heart. (B) TTX has no effect on the muscle activity. Arrow head: Perfusing solution was changed from normal saline to saline containing TTX $\left(10^{-6} M\right)$.

extends from the first to the twelfth thoracic segments and is suspended in the pericardial cavity. The heart is 6.4 to $8.6 \mathrm{~mm}$ in length, but less than $1 \mathrm{~mm}$ in width in the preparations used. The heart tube bifurcates near its anterior end and opens at the head region just under the eyes; its posterior end is closed. The heart has 11 ostia on each side, but no defined walled arteries are present. The heart wall is composed of a single layer of myocardial cells (Fig. 1C). Myofibrils are present in the outer half of the myocardial cell. No nerve cells were found in the heart.

Spontaneous membrane potential changes in the heart muscle

Electrical activity of the heart muscle and contractions of the heart were recorded simultaneously. As shown in Figure 2A, each heartbeat followed a periodic slow depolarizing potential of the heart muscle. The frequency and amplitude of the slow potential were not stable, but changed during measurement in the samc preparation. The frequency and amplitude of the slow potentials, measured about $30 \mathrm{~min}$ after isolation, were $188.2 \pm 32.9 /$ $\min ($ mean $\pm \mathrm{SD}, n=64$ ) and $17.5 \pm 6.3 \mathrm{mV}$ (mean \pm $\mathrm{SD}, n=64$ ), respectively. No spike potentials appeared on the slow potential. The maximum membrane potential between the slow potentials was $59.2 \pm 3.6 \mathrm{mV}$ (mean $\pm \mathrm{SD}, n=56$ ).
TTX arrests the beat of decapod crustacean hearts by suppressing neuronal spiking in the cardiac ganglion (e.g., Tazaki, 1971). However, the spontaneous slow potentials in the heart muscle of Triops were not affected by treatment with TTX at concentrations up to $10^{-6} M$ (Fig. 2B).

\section{Synchronous activity of the heart muscle cells}

Muscle activity was simultaneously recorded from two sites in the heart. Figure 3A shows two examples in which one recording site was near the anterior end (upper traces), and the other near the posterior end of the heart (lower traces). The periodic membrane potential changes were synchronized among the muscle cells within a time difference of less than $50 \mathrm{~ms}$ (A1, $16 \mathrm{~ms}$; A2, $12 \mathrm{~ms}$ ). Thus, the whole heart beat almost simultaneously.

In the case shown in Figure 3A1, the slow potential recorded from the anterior region preceded that recorded from the posterior region. But in the case shown in Figure $3 \mathrm{~A} 2$, the slow potential recorded from the posterior region preceded that recorded from the anterior region. No local region of the heart had activity that consistently preceded that of the rest of the organ.
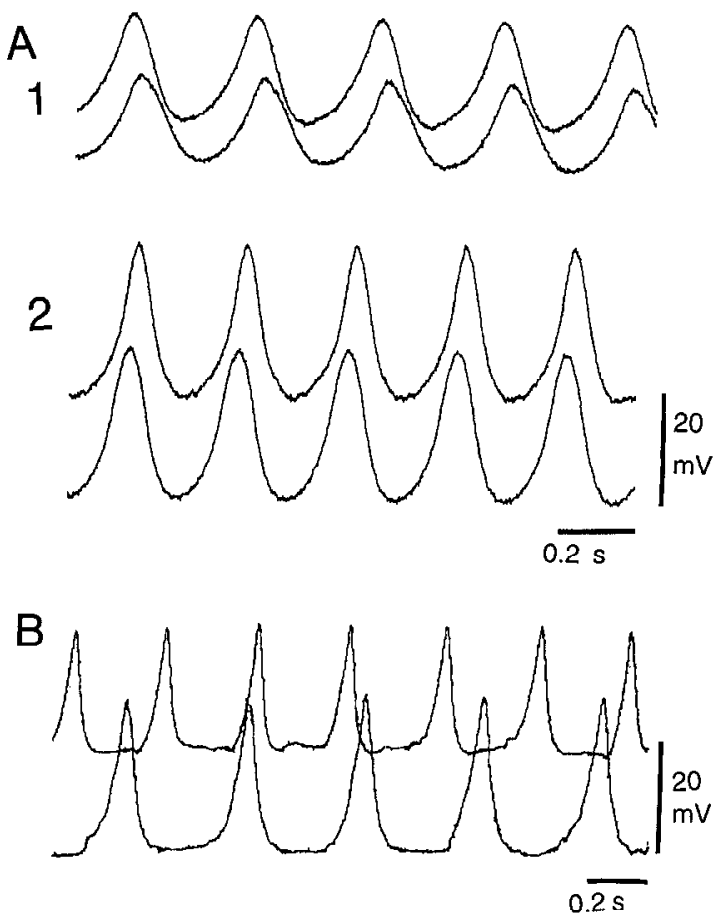

Figure 3. (A) Intracellular muscle activity recorded simultaneously from two sites in the heart: one near the anterior end (upper traces), and one near the posterior end (lower traces). Recordings 1 and 2 are from different preparations. (B) Intracellular muscle activity recorded simultaneously from two separated fragments of the same heart. The heart was cut transversely into four separated fragments. Reckoning from anterior to posterior, the records were from the first (upper trace) and the third (lower trace) fragments. 


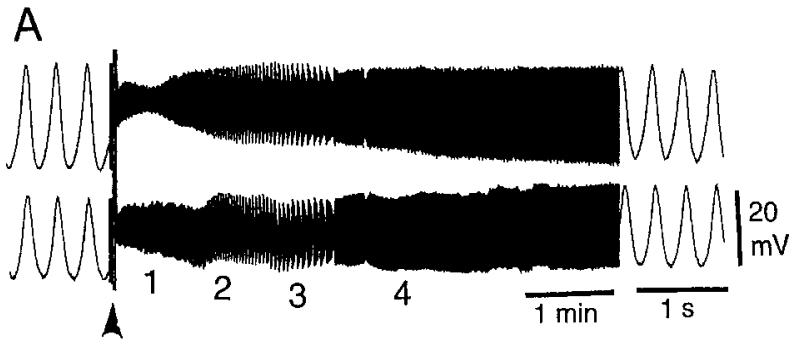

B
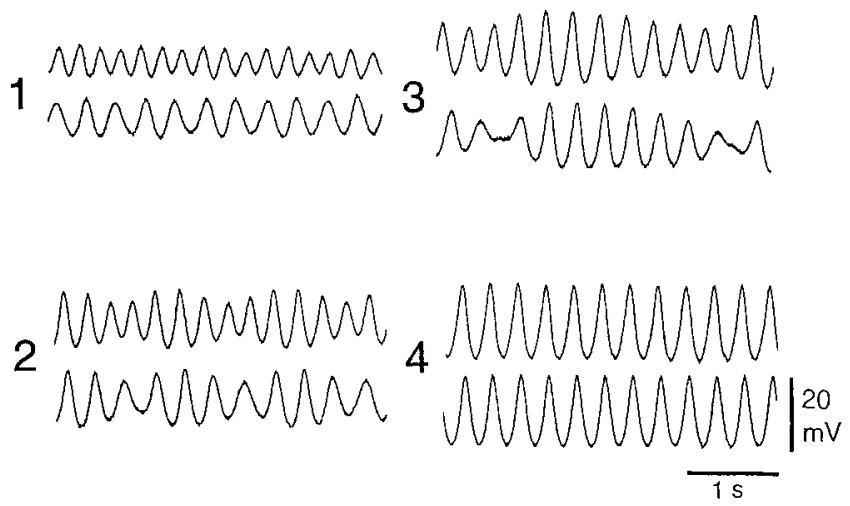

Figure 4. (A) Intracellular muscle activity recorded simultancously from two sites: near the anterior end (upper trace) and near the posterior end (lower trace) of the heart. Arrow head: A mechanical stimulus (touching) with a small glass rod was applied to the central region of the heart. (B) Records with a faster sweep. The number of each record corresponds to the number in $(\Lambda)$.

We next examined muscle activity in cut fragments of the heart. Figure 3B shows an example in which the heart was cut transversely into four separated fragments, and muscle activity was recorded simultaneously from two of them. Activity similar to that found in the intact heart was recorded from the two fragments, each with its own rhythm. Thus, a fragment isolated from any portion of the heart exhibited spontaneous rhythmic activity.

A mechanical stimulus applied to the heart by touching or pressing the heart wall with a small glass rod produced a transient systolic arrest within a localized region near the stimulated site. When the central region of the heart was arrested by the local application of a mechanical stimulus, the rhythmical activities in the anterior and the posterior regions of the heart gradually became synchronized. We therefore examined the time course for achieving synchronization of the muscle activities. The experiment in Figure 4 is exemplary. Before application of the stimulus (Fig. 4A, left portion), the slow potentials recorded from the anterior (upper trace) and posterior (lower trace) regions were synchronized at a frequency of $177 / \mathrm{min}$ with a time difference of about $35 \mathrm{~ms}$. Upon stimulation, the muscle cell membranes in both regions depolarized by more than $10 \mathrm{mV}$, and then recovered gradually to the control level (Fig. 4A, middle and right portions). After the stimulation (Fig. 4B), the frequency of the slow potential increased conspicuously $(270 / \mathrm{min})$ in the anterior region, but was almost unchanged in the posterior region (B1). Subsequently, the frequency and amplitude of the slow potential in both regions changed and the rhythm became irregular (B, 2 and 3); then the rhythm became synchronized as in the control condition (B4).

\section{Electrical coupling among the heart muscle cells}

Two electrodes, one for current injection and the other for recording, were inserted into muscle cells at different sites of the heart. In the experiment of Figure 5A, the
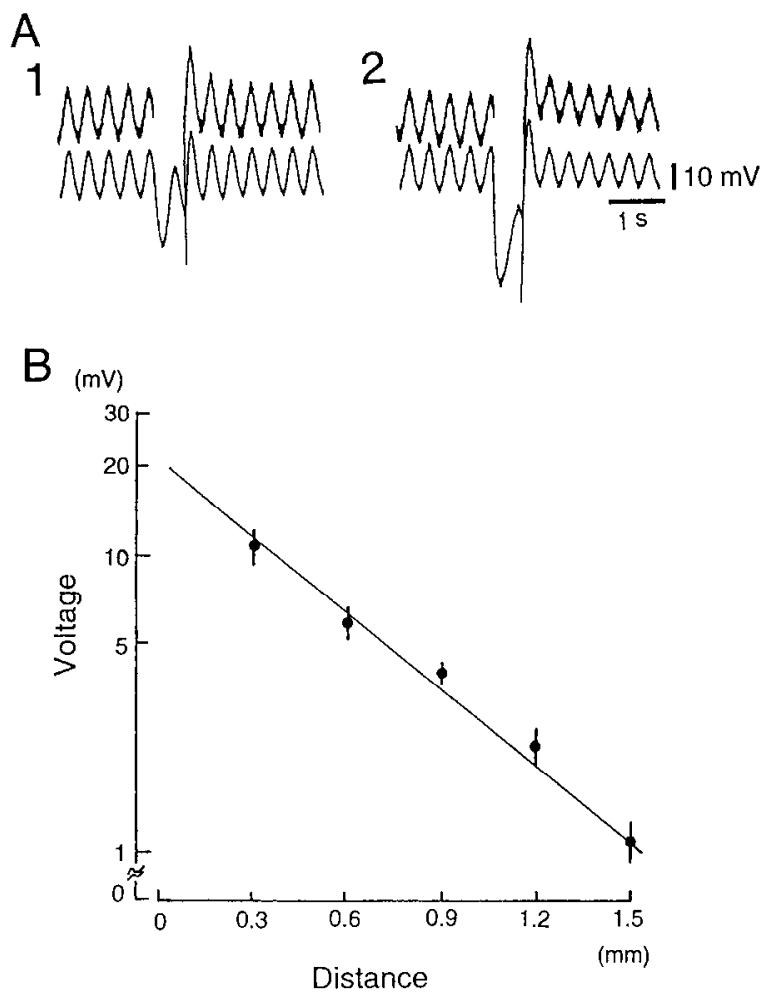

Figure 5. Electrical coupling among the heart muscle cells. $(\Lambda)$ Intracellular muscle activity recorded simultaneously from two sites in the heart. Distance between the two electrodes was about $600 \mu \mathrm{m}$. A hyperpolarizing current pulse, $500 \mathrm{~ms}$ in duration, was injected into the heart muscle through one of the two electrodes, using a bridge circuit (upper traces). Intensity of the current was $100 \mathrm{nA}$ in Al and $200 \mathrm{nA}$ in A2. (B) Relationship, in an arrested heart, between the amplitude of the electrotonic potential and the distance between the current and the recording electrodes. A hyperpolarizing current pulse (100 ms, $80 \mathrm{nA})$ was injected into the heart muscle through the current electrode, and the resultant electrotonic potential was recorded at various distances from the current electrode. The amplitude of each electrotonic potential was plotted on a logarithmic scale against each distance from the current electrode. Mean values $( \pm$ SEM) of 5 measurements in the same heart were plotted. 
two electrodes were about $600 \mu \mathrm{m}$ apart. When a hyperpolarizing current pulse $(500 \mathrm{~ms}, 100 \mathrm{nA})$ was injected, the membrane of the muscle cell hyperpolarized (A1). The amplitude of the hyperpolarization increased with the intensity of the current injected (A2, $200 \mathrm{nA})$.

The spread of electrotonic potentials was examined with heart preparations that had stopped beating after perfusion for several hours. The electrotonic potential produced by injecting a hyperpolarizing current pulse ( $100 \mathrm{~ms}, 80 \mathrm{nA}$ ) was recorded while the distance between the current and recording electrodes, along the ventral longitudinal midline of the heart, was varied. The amplitude of the hyperpolarization decreased with increasing distance between the two electrodes. A semi-logarithmic plot of the amplitude of hyperpolarization against the distance between the current and the recording electrode shows the amplitude decreasing linearly with the distance (Fig. 5B). The length constant $(\lambda)$ calculated from the relationship was $0.55 \pm 0.08 \mathrm{~mm}$ (mean $\pm \mathrm{SD}, n=5$ ).

\section{Effects of current injection on the muscle activity}

A brief depolarizing or hyperpolarizing current pulse $(50 \mathrm{~ms}, 50 \mathrm{nA})$ was injected into the heart muscle at various phases of the rhythmical activity. The inter-peak interval was defined as the time between the peaks of two slow potentials. The phase of the applied current pulse (stimulation phase) was defined as the interval between the peak of the slow potential preceding the pulse and the onset of the pulse; it was taken as a percentage of the preceding inter-peak interval (control inter-peak interval). The resultant phase shift of subsequent beat cycles was measured at the peak of the third slow potential from the applied stimulus; it was taken as a percentage of the control inter-peak interval.

As shown in Figure 6A, a depolarizing pulse applied at $27 \%$ produced a phase delay (A1), and a pulse applied at $64 \%$ produced a phase advance in the subsequent beat cycles (A2). Figure $6 \mathrm{~B}$ shows the relationship between the phase shift and the stimulation phase (phase-response curve). The curve was biphasic at a stimulation phase of approximately $40 \%$ : a stimulus applied at an earlier phase produced a phase delay $(+)$, and a stimulus applied at a later phase produced a phase advance $(-)$. In contrast, as shown in Figure 7A, a hyperpolarizing pulse applied at $36 \%$ produced a phase advance, and one applied at $85 \%$ produced a phase delay in the subsequent beat cycles. The phase response curve was biphasic at a stimulation phase of approximately $50 \%$ : stimuli applied at an earlier phase produced a phase advance (-), and stimuli applied at a later phase produced a phase delay (+) (Fig. 7B).

We next examined the effects on the muscle activity of injecting repeated brief current pulses. A brief depolarizing or hyperpolarizing current pulse $(50 \mathrm{~ms}, 50 \mathrm{nA})$ was

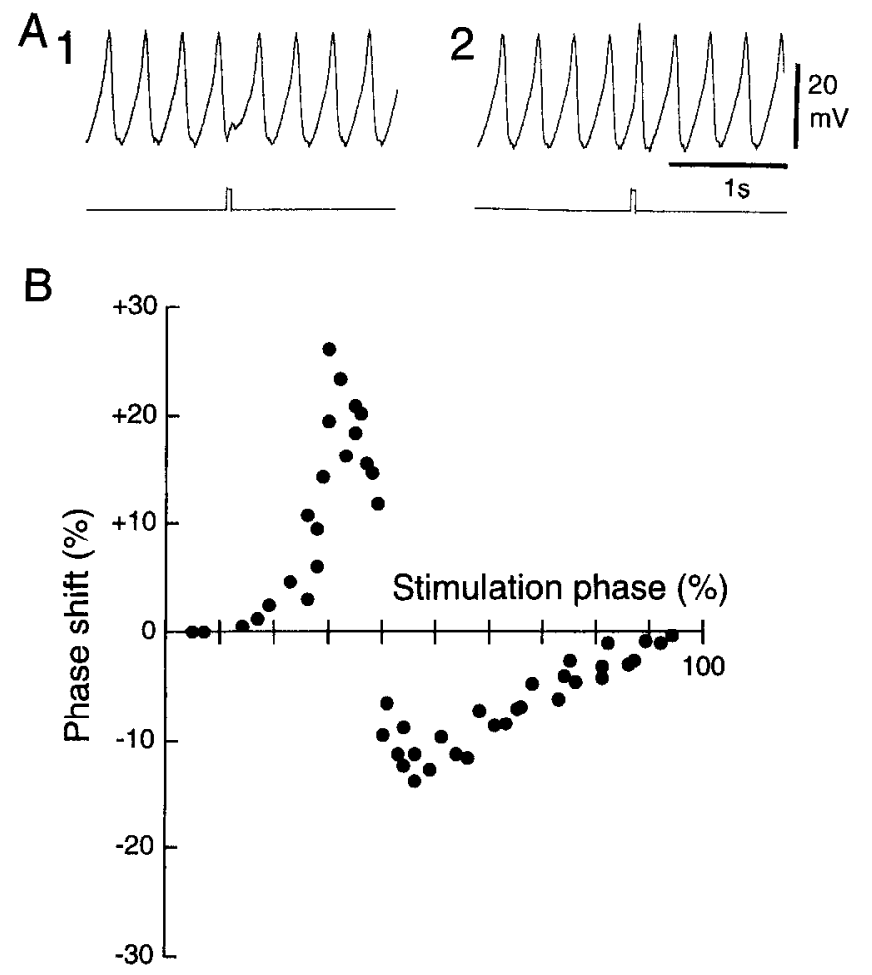

Figure 6. Effects on the muscle activity of injecting a brief depolarizing current pulse. (A) Each record shows the intracellular activity of the heart muscle (upper trace) and a monitor of the stimulus (lower trace). The recording electrode was always inserted near the anterior end of the heart, and the current electrode near the posterior end. A brief depolarizing current pulse $(50 \mathrm{~ms}, 50 \mathrm{nA})$ was applied at various phases (stimulation phase) of the inter-peak interval. See text for definitions of the stimulation phase and the inter-peak interval. Stimulation phases were $27 \%$ (1) and $64 \%$ (2). (B) Relationship between phase shift and stimulation phase (phase-response curve). Fifty brief depolarizing pulses were applied at various stimulation phases. Each phase delay $(+)$ or phase advance $(-)$ was plotted against each stimulation phase.

injected into the heart muscle at various frequencies. For example (Fig. 8), muscle activity at a control frequency of $150 / \mathrm{min}$ could be entrained to a frequency $17 \%$ lower (A1), or $13 \%$ higher (A2), by application of repeated brief depolarizing pulses. Similarly, muscle activity could be entrained to a frequency $19 \%$ lower (B1), or $13 \%$ higher (B2), than the control by application of repeated brief hyperpolarizing pulses.

We examined effects of injecting, for $10 \mathrm{~s}$, depolarizing or hyperpolarizing DC current of various intensities into the heart muscle. Figure 9 shows an example. The frequency of slow potentials increased slightly $(\mathrm{A},+2.1 \%)$ when depolarizing $\mathrm{DC}$ current $(10 \mathrm{nA})$ was applied. The effect increased $(\mathrm{B},+6.8 \% ; \mathrm{C},+9.6 \%)$ with the intensity of applicd current (B, $20 \mathrm{nA} ; \mathrm{C}, 30 \mathrm{nA})$. By contrast, hyperpolarizing $\mathrm{DC}$ current $(\mathrm{D}, 40 \mathrm{nA})$ decreased $(-13.0 \%)$ the frequency of the slow potentials, and the decrease was larger $(\mathrm{E},-17.1 \%)$ as the intensity of the 

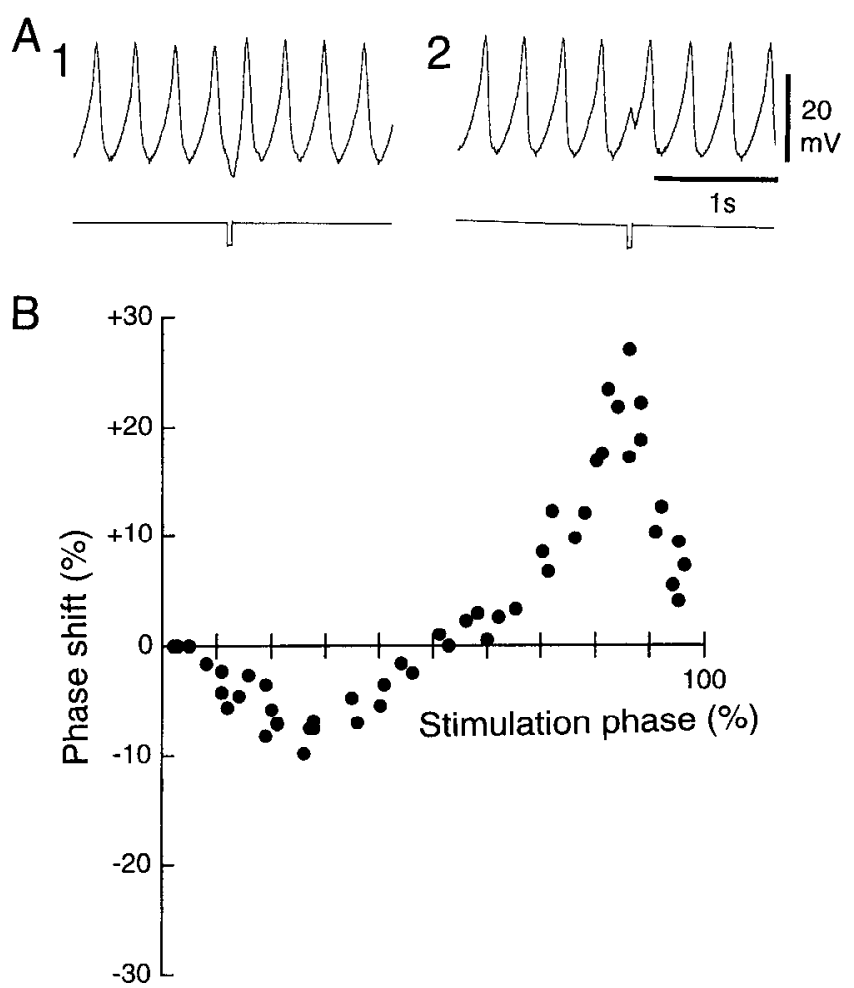

Figure 7. Effects on the muscle activity of injecting a brief hyperpolarizing current pulse. (A) Each record shows the intracellular activity of the heart muscle (upper trace) and a stimulus monitor (lower trace). The recording electrode was inserted near the anterior end of the heart, and the current electrode near the posterior end. A brief hyperpolarizing current pulse (50 ms, $50 \mathrm{nA}$ ) was applied at various phases (stimulation phase) of the inter-peak interval. See text for definitions of the stimulation phase and the inter-peak interval. Stimulation phases were $36 \%$ (1) and $85 \%$ (2). (B) Relationship between phase shift and stimulation phase (phase-response curve). Fifty brief hyperpolarizing pulses were applied at various stimulation phases. Each phase delay $(+)$ or phase advance $(-)$ was plotted against each stimulation phase. From the same preparation as in Fig. 6.

applied current increased (E, $50 \mathrm{nA})$. Hyperpolarizing DC current of high intensity (F, $60 \mathrm{nA}$ ) reduced the amplitude of the slow potential markedly, causing it to disappear. Figure 10 shows the relationship between the frequency change of slow potential and the intensity of current applied. The relationship was almost linear, but hyperpolarization with a DC current more intense than a critical value (50 to $120 \mathrm{nA}$ in 8 preparations) suppressed the slow potentials, and arrested the heart.

The effects of injecting current at various sites on the heart were examined. In Figure 11, for example, the muscle activity was recorded simultaneously near the anterior (upper traces) and the posterior ends (lower traces) of the heart. Muscle activity was suppressed completely by the injection of a hyperpolarizing DC current $(5 \mathrm{~s}, 100 \mathrm{nA})$ through the anterior clectrode (A) or through the posterior electrode (B). Thus, regardless of the site of current injec- tion in the heart, the same effects on the muscle activity were induced.

\section{Discussion}

Branchiopod hearts lack a cardiac ganglion

The heart of Triops longicaudatus consists of a single layer of myocardial cells. Myofibrils are found in the outer half of these cells (Fig. 1C). Electron microscopic observations on the heart of another tadpole shrimp, Lepidulus arcticus, showed that the heart consists of a single layer of strongly polarized myocardial cells, each with their myofibrillar part facing the epicardium (Tjønneland, et al., 1980). The myofibrils cross each other in the cell and form a contractile network. Tjønneland et al. also found intercalated discs between adjacent myocardial cells in the region of the myofibrils.

No nerve cells were found in the heart of Triops by

\section{A \\ 1}

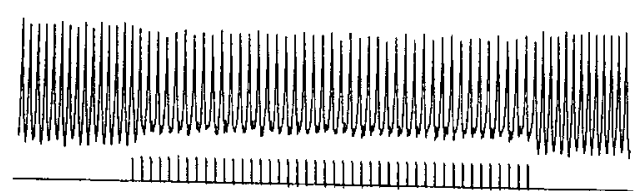

2

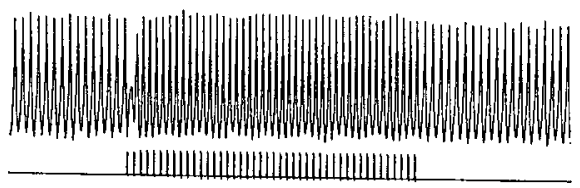

B

1
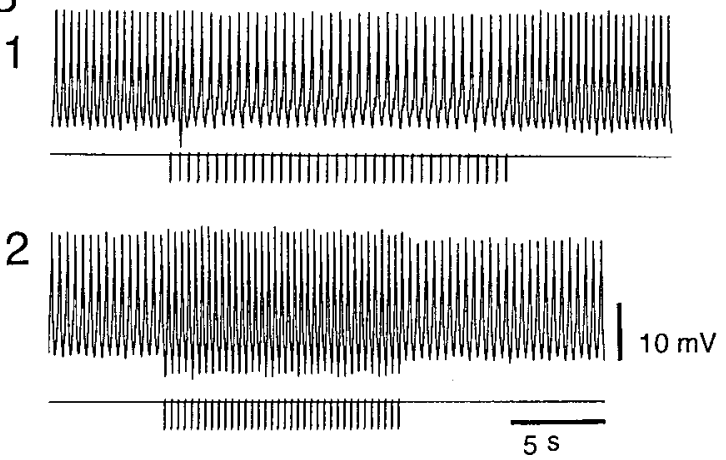

Figure 8. Effects on muscle activity of injecting repeated brief current pulses. Intracellular activity of the heart muscle (upper trace) and a stimulus monitor (lower trace) are shown in each record. The recording electrode was inserted near the anterior end of the heart, and the current electrode near the posterior end. (A) A brief depolarizing current pulse $(50 \mathrm{~ms}, 100 \mathrm{nA})$ was repeatedly applied at frequencies of $124 / \mathrm{min}$ in $A 1$, and $169 / \mathrm{min}$ in $A 2$. Frequency of the muscle activity under nonstimulated conditions (control) was $150 / \mathrm{min}$. (B) A brief hyperpolarizing current pulse $(50 \mathrm{~ms}, 100 \mathrm{nA})$ was repeatedly applied at frequencies of $122 / \mathrm{min}$ in $\mathrm{B} 1$, and at $169 / \mathrm{min}$ in B2. Control frequency of the muscle activity was $155 / \mathrm{min}$. From the same preparation as in Figs. 6 and 7. 


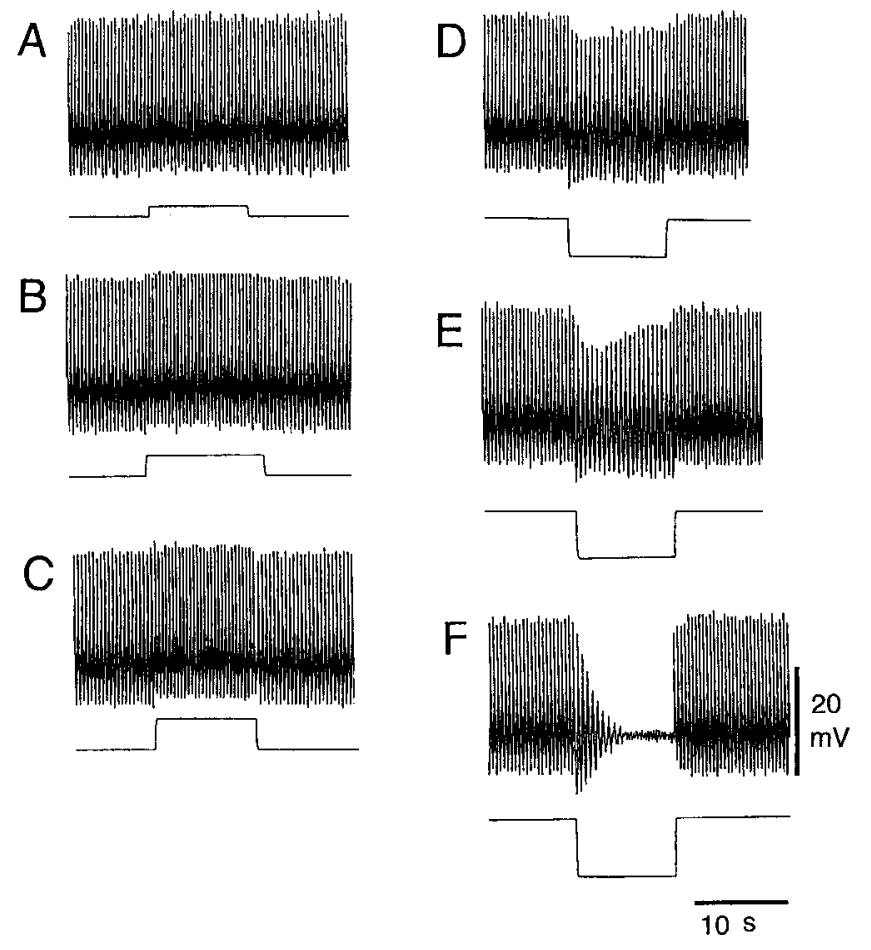

Figure 9. Effects on myocardial activily of injecting DC current. The recording electrode was inserted near the anterior end of the heart, and the current electrode near the posterior end. In each record, intracellular activity of the heart muscle (upper trace) and monitor of the current applied (lower trace) are shown. Depolarizing (+) or hyperpolarizing $(-)$ DC current was applied for $10 \mathrm{~s}$. Intensity of the current (nA) was $+10(\mathrm{~A}),+20(\mathrm{~B}),+30(\mathrm{C}),-40(\mathrm{D}),-50(\mathrm{E})$, and $-60(\mathrm{~F})$.

vital staining with methylene blue or by microscopic observation of serial sections of the heart. Ingle (cited by Prosser, 1942) also used various nerve-staining methods, but failed to find nerve cells in the heart of Daphnia. Furthermore, although electron microscopic methods have been used to study the hearts of several branchiopods-Daphnia (Stein et al., 1966), Lepidurus (Tjønneland et al., 1980), Branchinecta, Artemia, Branchipus, and Streptocephalus (Økland et al., 1982)-no neural elements have ever been reported. It is unlikely that the heart of branchiopods has a cardiac ganglion.

\section{Myogenic activity}

The heartbeat of Triops is associated with periodic slow depolarizing potentials in the myocardium (Fig. 2A). This activity was not affected by application of TTX (Fig. 2B). In the neurogenic heart of crustaceans, the heart muscle has no endogenous rhythmicity and is driven by a cardiac ganglion (reviewed by Maynard, 1960; Prosser, 1973). The heart of these animals stops beating if the cardiac ganglion is removed (reviewed by Krijgsman, 1952) or if TTX is applied, suppressing neuronal spiking in the cardiac ganglion (Anderson and Cooke, 1971). In the myogenic heart of embryos and early juveniles of the isopod Ligia, the muscle activity is not affected by application of TTX, except for the TTX-sensitive spike on the slow potential (Yamagishi, 1996; Yamagishi and Hirose, 1997). The ineffectiveness of TTX on the Triops heart supports the idea that the rhythmicity of this myocardium is generated endogenously.

The electrical activity of the Triops heart consists of only slow potentials, and the amplitude of the slow potential changes gradually during measurement. But no spike potentials ever appeared, even after termination of a hyperpolarizing DC current injected into the heart muscle (e.g., Fig. 8). The heart muscle of Triops, generating membrane potential oscillation, may lack the ability to generate all-or-none membrane potentials.

\section{Characteristics of the heart as a single muscle oscillator}

No localized regions of the Triops heart consistently generated potentials that preceded those of other regions (Fig. 3A). Moreover, any cut fragment of the heart exhibited spontaneous muscle activity (Fig. 3B). These results suggest that the heart of Triops has diffuse myogenicity, and that individual muscle cells are inherently oscillatory. When the central region of the heart was arrested by a mechanical stimulus, the anterior and posterior regions

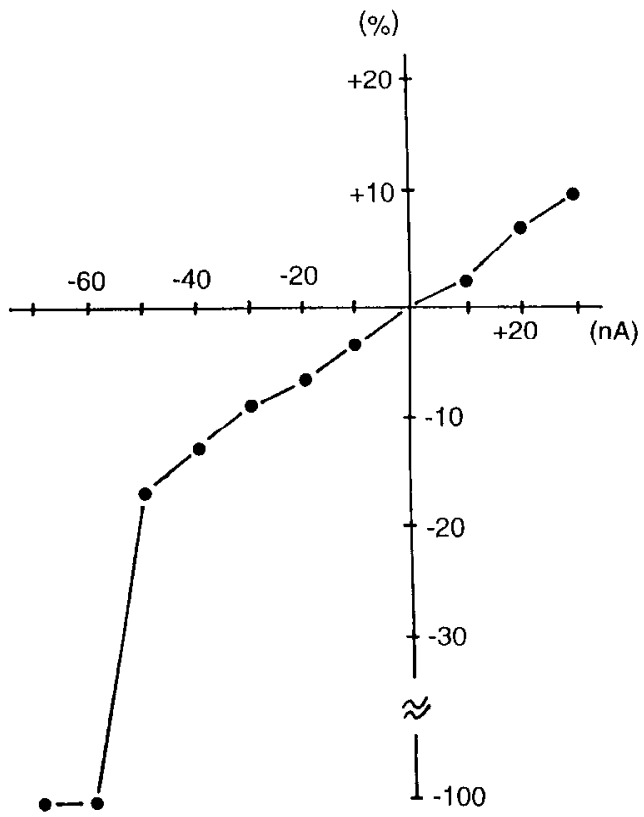

Figure 10. Relationship between the change of frequency of the muscle activity and the intensity of the current applied. Each percentage change in the slow potential frequency of the muscle activity was plotted against the intensity (nA) of the depolarizing (+) ог hyperpolarizing $(-)$ current applied. From the same preparation as in Fig. 9. 
A
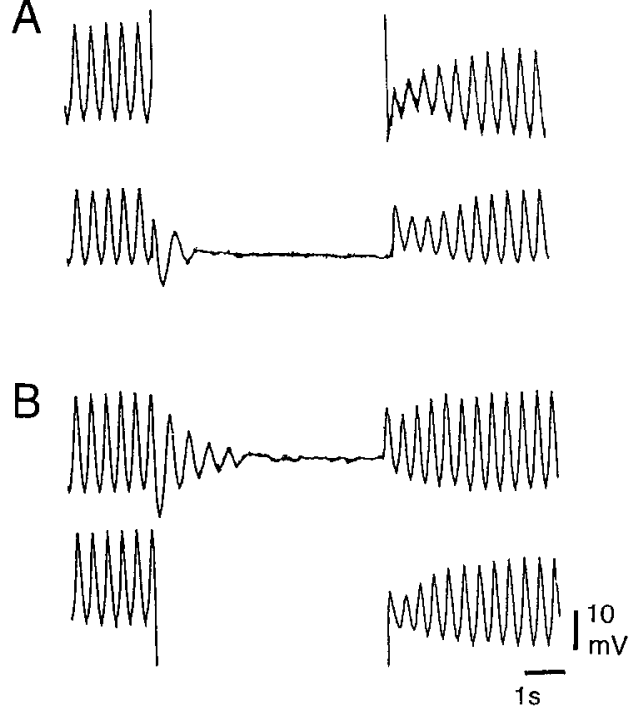

Figure 11. Effects on myocardial activity of current injection at two siles of the heart. Intracellular activity of the heart muscle was recorded simultaneously at two sites in the heart: one near the anterior end (upper traces), and one near the posterior end (lower traces). (A) Hyperpolarizing DC current (100 nA) was injected for $5 \mathrm{~s}$ through the electrode inserted at the anterior region, using a bridge circuit. (B) Hyperpolarizing DC current ( $100 \mathrm{nA})$ was injected for $5 \mathrm{~s}$ through the electrode inserted at the posterior region, using a bridge circuit.

beat independently with their own rhythms; subsequently their rhythms were gradually resynchronized (Fig. 4). This suggests that the heart muscle cells synchronize their activities through interaction. The high sensitivity of the Triops heart to mechanical stimuli suggests some stretchmediated contribution to the synchronization of the muscle cells. However, spontaneous activity was rapidly synchronized (Fig. 3A), and the heart muscle cells appear to be electrically coupled (Fig. 5).

We therefore conclude that the heart muscle cells are synchronized through electrical interactions, as in the myogenic hearts of other invertebrate species (reviewed by Ebara, 1993). The heart of Triops can be said to have a diffuse myogenic pacemaker with the properties of a functional syncytium. These characteristics of the heart are very similar to those of the myogenic hearts of embryos and early juveniles of the isopod Ligia (Yamagishi and Hirose, 1997) and of most molluscs (reviewed by Hill and Welsh, 1966; Irisawa, 1978; Jones, 1983).

The rhythm of the muscle activity could be phaseshifted by a brief current pulse (Figs. 6 and 7) and could be entrained to the frequency of repeated, brief current pulses injected into the muscle cell (Fig. 8). In addition, the frequency of the muscle activity changed linearly with the intensity of the injected DC current, and the muscle activity was suppressed completely during the injection of strong hyperpolarizing DC current (Figs. 9 and 10).
These responses to external inputs are characteristic of endogenous oscillators in general (e.g., Pavlidis, 1973). Moreover, these effects on muscle activity could be induced by current injection at any site in the heart (Fig. 11). Thus, the heart of Triops can be regarded as a single muscle oscillator.

\section{Diversity in the pacemaker mechanism of crustacean hearts}

Many investigations on the heartbeat pacemaker mechanism of crustaceans, mainly of decapods, have led to the generalization that crustacean hearts are neurogenic, with the cardiac ganglion acting as the dominant pacemaker (reviewed by Krijgsman, 1952; Maynard, 1960; Prosser, 1973). Recently, however, Yamagishi and Hirose (1997) showed that the cardiac pacemaker in the isopod Ligia exotica is transferred from the heart muscle to the cardiac ganglion during juvenile development. Now we have shown that the heartbeat of the branchiopod Triops longicaudatus is myogenic, and that the heart muscle acts as a diffuse pacemaker. Clearly, the heartbeat pacemaker mechanism in crustaceans is more diverse than previously thought.

\section{Acknowledgments}

We thank Dr. K. Sekiguchi for his kind support throughout this study. We also thank Dr. K. Oami for his helpful discussion, and Dr. Darryl Macer for his reading of the manuscript. This work was supported in part by a Grant-in-Aid (No. 0864057) from the Ministry of Education, Science, Sports and Culture of Japan to H.Y.

\section{Literature Cited}

Anderson, M., and I. M. Cooke. 1971. Neural activation of the heart of the lobster, Homarus americanus. J. Exp. Biol. 55: 449468.

Baylor, E. R. 1942. Cardiac pharmacology of the cladoceran, Daphnia. Biol. Bull. 83: 165-172.

Bekker, J. M., and B. J. Krijgsman. 1951. Physiological investigations into the heart function of Daphnia. J. Physiol. 115: 249257.

Ebara, A. 1993. Self-control of heart rhythm in some invertebrates. Pp. 1-9 in Trends in Comparative Biochemistry and Physiology 1, J. Menon, ed. Council of Scientific Research Integration, Trivandrum, India.

Greenberg, M. J. 1965. A compendium of responses of bivalve hearts to acetylcholine. Comp. Biochem. Physiol. 14: 513-539.

Hill, R. B., and J. H. Welsh. 1966. Heart, circulation and blood cells. Pp. 125-174 in Physiology of Mollusca, K. M. Wilbur and C. M. Yonge, eds. Academic Press, New York.

Horne, F. R. 1966. Some aspects of ionic regulation in the tadpole shrimp Triops longicaudatus. Comp. Biochem. Physiol. 19: 313316.

Irisawa, H. 1978. Comparative physiology of the cardiac pacemaker mechanism. Physiol. Rev. 58: 461-498. 
Jones, H. D. 1983. The circulatory system of gastropods and bivalves. Pp. 189-235 in The Mollusca, A. S. M. Saleuddin and C. M. Yonge, eds. Academic Press, New York.

Krijgsman, B. J. 1952. Contractile and pacemaker mechanisms of the heart of arthropods. Biol. Rev. 27: 320-346.

Maynard, D. M. 1960. Circulation and heart function. Pp. 161-226 in The Physiology of Crustacea 1, T. H. Watermann, ed. Academic Press, New York.

Økland, S., A. Tjønneland, L. N. Larsen, and A. Nylund. 1982. Heart ultrastructure in Branchinecta paludosa, Artemia salina, Branchipus shaefferi, and Streptocephalus sp. (Crustacea, Anostraca). Zoomorphology 101: 71-81.

Pavlidis, T. 1973. Bivlogical Oscillators: Their Mathematical Analysis. Academic Press, New York.

Prosser, C. L. 1942. An analysis of the action of acetylcholine of hearts, particularly in arthropods. Biol. Bull. 83: 145-164.

Prosser, C. L. 1973. Circulation of body fluids. Pp. 822-856 in Compara tive Animal Physiology, C. L. Prosser, ed. Saunders, Washington, DC.
Stein, R. J., W. R. Richter, R. A. Zussman, and G. Brynjolfsson. 1966. Ultrastructural characterization of Daphnia heart muscle. $J$. Cell. Biol. 29: 168-170.

Tazaki, K. 1971. The effects of tetrodotoxin on the slow potential and spikes in the cardiac ganglion of a crab, Eriocheir japonicus. Jpn. J. Physiol. 21:529-536.

Tjønneland, T., B. Midttun, S. Økland, and H. O. Liebich. 1980. Heart ultrastructure in Lepidurus arcticus Pallas (Crustacea, Branchiopoda, Notostraca). Cell Tiss. Res. 212: 203-212.

Yamagishi, H. 1996. Endogenous oscillatory activity and TTX-sensitive spikes of the heart muscle in early juveniles of the isopod crustacean Ligia exotica. Experientia 52: 583-586.

Yamagishi, II., and E. Hirose. 1997. Transfer of the heart pacemakcr during juvenile development in the isopod crustacean Ligia exotica. J. Exp. Biol. 200: 2393-2404.

Yamagishi, H., H. Ando, and T. Makioka. 1993. The heart beat of Triops longicaudatus (Crustacea, Branchiopoda) is myogenic. Zool. Sci. 10, Supple. 95. 